\title{
Conclusion
}

Des laits individuels de vaches de race hollandaise de la région de Rennes, ayant des teneurs en matière grasse de 34,5 à $38 \mathrm{~g}$ par litre, ont une tension superficielle de 47,5 à $48 \mathrm{dyn} / \mathrm{cm}$ au tensiomètre de Lecomte du Nouy pour des mesures à 20-220 C. Dans les conditions adoptées, les résultats peuvent varier de plus ou moins $0,2 \mathrm{dyn} / \mathrm{cm}$ autour de la moyenne.

Le mouillage abaisse la tension superficielle jusqu'à un minimum d'environ $41 \mathrm{dyn} / \mathrm{cm}$ pour des mouillages de 20 à 40 volumes d'eau pour 100 volumes de mélange. Au-delà de 40 p. 100, la tension se relève. Pour de très fortes dilutions aqueuses, la tension se rapproche de la tension du lait pur sans l'atteindre.

Avec des laits récemment traits, ou correctement gardés quelques jours, en tout cas non altérés, un faible mouillage abaissant la tension superficielle d'une façon appréciable, cette mesure pourraît être prise en considération pour confirmer les analyses d'un lait suspect de fraude par mouillage.

La tension superficielle d'un lait concentré non sucré, homogénéisé, ayant 7 p. 100 de matière grasse, est de 52 à $53 \mathrm{dyn} / \mathrm{cm}$ à $22,5^{\circ}$ C. Le mouillage diminue la tension superficielle jusqu'à $49 \mathrm{dyn} / \mathrm{cm}$. Elle se relève légèrement pour de très fortes dilutions, sans dépasser $50 \mathrm{dyn} / \mathrm{cm}$.

\section{L'UTILISATION DE COLORANTS \\ POUR déceler LA PRÉsence d'ANTIBIOTIQUES DANS LE LAIT (1)}

\author{
par \\ P. KASTLI
}

\section{I. - Introduction}

Les rapports établis précédemment par le Pr STORGaRDs pour la commission F.I.L. de la Production du Lait et publiés dans le Bulletin de la Fédération en 1962 (partie III), traitent de façon complète et détaillée des procédés mis en œuvre pour déceler la présence d'antibiotiques dans le lait. On dispose actuellement de

(1) Bull. F.I.L., 1963, VI (Rapport présenté par M. le Professeur Docteur P. Kästli et reproduit avec l'autorisation de la Fédération Internationale de Laiterie). 
méthodes permettant de contrôler, dans un temps relativement court et avec une sécurité suffisante, une fourniture de lait dans laquelle se trouvent des antibiotiques. D'après les renseignements qui nous sont parvenus des Etats-Unis et du Danemark, il a été ainsi possible de réduire dans une proportion considérable le pourcentage du lait commercialisé contenant des antibiotiques. Kosıkowski, signale par exemple que, grâce à un contrôle régulier du lait livré aux laiteries, le pourcentage des échantillons renfermant des antibiotiques est tombé de 6,5 à 0,45 [14].

Quoi qu'il en soit, des efforts doivent être poursuivis pour que le contrôle du lait livré aux laiteries comprenne, aussi souvent que possible, la recherche des antibiotiques.

Un tel contrôle ne peut toutefois être effectué que lorsque la livraison du lait est relativement centralisée et que, par conséquent, les opérations peuvent s'effectuer en laboratoire. Cependant, dans de nombreuses régions laitières, de petites fermes livrent le lait aux centres locaux de ramassage et à de petites usines (par exemple à des fromageries). Il est pratiquement impossible, dans ces entreprises, de contrôler à intervalles réguliers - et moins encore quotidiennement - la présence d'antibiotiques dans le lait, car cela entraînerait des dépenses trop élevées. D'ailleurs, le problème des frais de contrôle en laboratoire se pose non seulement dans les petites entreprises, mais aussi dans les laiteries qui reçoivent d'importantes quantités de lait.

En outre, lorsque le lait livré aux laiteries provient de centres ruraux de ramassage, une nouvelle difficulté intervient au moment du contrôle effectué à la laiterie : e'est un lait de mélange et il n'est pas possible de localiser l'origine de la contamination par les antibiotiques.

A l'absence d'un contrôle régulier et effectué peu après la traite du lait fourni aux centres de ramassage et aux petites entreprises de transformation par les différentes fermes, s'ajoute le fait que le délai qui s'écoule entre la livraison du lait et son transport ou sa transformation ultérieure demeure trop court pour que le lait contenant des antibiotiques puisse être éliminé. Les progrès considérables réalisés dans les méthodes de contrôle, qui permettent d'obtenir déjà des résultats après trois ou quatre heures, n'ont rien changé à cet état de choses.

Dans ces conditions, le seul contrôle possible du lait donnant un résultat sûr, consiste à déceler la présence des antibiotiques par un "test sensoriel ". Nous entendons par là une 'odeur facilement reconnaissable ou un changement de couleur. L'addition d'une substance odorante ne paraît guère de nature à donner des résultats 
pratiques; en revanche, on a procédé à des essais sur une très grande échelle par addition de colorants.

Cette adjonction doit, en principe, répondre aux impératifs ci-après :

1. Le colorant doit persister dans la préparation aussi longtemps que l'antibiotique;

2. Il ne doit pas être toxique pour le consommateur de lait ou de produits laitiers ;

3. Il ne doit pas irriter le tissu mammaire ;

4. Il ne doit pas nuire à l'efficacité de l'antibiotique ;

5. Son élimination doit se faire parallèlement à celle de l'antibiotique, ou bien le colorant doit demeurer dans le lait aussi longtemps que l'antibiotique.

6. La coloration du lait doit être suffisamment intense pour demeurer visible même après une dilution importante avec $\mathrm{du}$ lait normal.

On voit que les conditions que doivent remplir les colorants pour pouvoir être utilisés en limitent sensiblement le choix. Il est donc compréhensible que, jusqu'à présent, il n'ait été possible de retenir que quelques produits susceptibles d'être ajoutés à des préparations contenant des antibiotiques et destinées à des traitements intra-mammaires.

L'étude de la documentation technique ainsi que les renseignements recueillis et les expériences auxquelles a procédé le rapporteur lui-même, ont permis d'aboutir aux constatations ciaprès en ce qui concerne les résultats des esssais effectués juqu'à présent.

\section{II. - Essais portant sur l'addition de colorants à des anti- biotiques}

En 1950, Hargrove [9] a procédé à un premier essai à l'aide de Bordeaux S. Il est apparu que l'élimination du colorant avec le lait était trop rapide et qu'elle n'allait pas de pair avec celle des antibiotiques.

Plus tard, en 1958, Hargrove, Lehman et Matthews [9] ont examiné d'autres substances et obtenu avec celles-ci les résultats ci-après :

La méthyl-4-ombelliferone s'est avérée impropre à cet usage parce qu'elle n'est visible qu'en lumière ultra-violette et a une action irritante sur le tissu glandulaire.

L'anthranilate de méthyle n'est parfaitement visible, lui aussi 
qu'en lumière ultra-violette. De plus, il est résorbé en partie dans la mamelle et est éliminé avec l'urine.

On a considéré que la chlorophylle liposoluble ne convenait pas en raison de la trop faible coloration qu'elle donne au lait.

La fluorescéine a donné de bons résultats surtout sous une forme soluble dans l'alcool, mais moins sous la forme liposoluble. La fluorescéine de sodium (uranine) a donné également des résultats satisfaisants.

L'esculine produit une fluorescence bleuâtre et permet également d'espérer des résultats favorables.

Dans ces expériences, on a utilisé soit $250 \mathrm{mg}$ de colorant pour $100000 \mathrm{U}$ de pénicilline, soit $400 \mathrm{~g}$ de colorant pour $300000 \mathrm{U}$ de pénicilline.

\subsection{Essais au moyen de la fluorescéine.}

Dans leurs expériences ultérieures (1959), HaRgrove, Plowman et WRIGHT [10] se sont bornés, compte tenu des essais dont il vient d'être question, à utiliser une préparation composée de $250 \mathrm{mg}$ de fluorescéine liposoluble et de $125 \mathrm{mg}$ d'uranine pour $100000 \mathrm{U}$ de pénicilline. Avec cette préparation, l'élimination du colorant allait de pair, dans une large mesure, avec celle de l'antibiotique. La coloration du lait provenant de la mamelle traitée était visible à l'œil nu durant une période allant jusqu'à 48 heures et, à la lumière ultraviolette jusqu'à 96 heures. L'essai effectué sur les animaux n'a pas permis de déceler un effet toxique. Par contre, on a constaté un certain affaiblissement de l'action de la pénicilline, de la dihydrostreptomycine, de la bacitracine, de la néomycine, de la polymycine, de l'ěrythromycine et de l'auréomycine, après un stockage des préparations.

En 1961, OBIGER, [16], a également fait des essais avec la fluorescéine, au cours d'expériences pour lesquelles il utilisait des préparations de divers antibiotiques, additionnées de $250 \mathrm{mg}$ ou de $500 \mathrm{mg}$ de colorant. Il a constaté que, si l'on ajoute de la fluorescéine ou de l'uranine, la modification de la couleur reste visible à l'œil nu pendant deux jours après le traitement et, en lumière ultraviolette, pendant trois jours. A l'œil nu, la coloration était encore décelable lorsque le lait était dilué dans la proportion de 1 à 100000 et, en lumière ultraviolette, de 1 à 1000000 .

D'autres essais avec la fluorescéine ont été effectués par Dawson et Fegan [4]. Ceux-ci ont estimé que ce colorant ne convenait pas parce qu'il est trop peú visible dans le lait (couleur jaune) et, qu'en outre, après un stockage de la solution colorée, on note une baisse de l'action de la pénicilline pouvant atteindre 50 p. 100. 


\subsection{Essais au moyen de chlorophylle.}

Smitasiri, Kosikowsi, GUthrie et Fincher [22] ont fait des essais au moyens de rocou, de cochenille, de chlorophylle soluble dans l'eau et de chlorophylle liposoluble. De tous ces colorants, seule la chlorophylle répondait aux exigences fondamentales. Le rocou et la cochenille ne convenaient pas parce que leur couleur ne différait pas assez de celle du lait et que leur élimination était trop rapide. Lors de leurs essais, les auteurs ont ajouté différentes concentrations de chlorophylle à $500000 \mathrm{U}$ de pénicilline. Une dose de $0,5 \mathrm{ml}$ de chlorophylle liposoluble par quartier a donné une coloration persistante pendant 5 traites, une dose de $1 \mathrm{ml}$ de chlorophylle, une coloration persistante pendant 10 traites (cinq jours). Si l'on ajoutait $10 \mathrm{ml}$ de chlorophylle liposoluble à l'antibiotique, le lait de la première traite suivant le traitement apparaissait encore coloré après dilution à $1 / 300$. Si, par contre, l'on utilisait seulement $5 \mathrm{ml}$ de chlorophylle, la coloration du lait n'était perceptible que jusqu'à une dilution de $1 / 7$. On a jugé que l'apparition de taches vertes sur le lait à la suite de l'emploi de chlorophylle liposoluble constituait un inconvénient. Ce phénomène a été également constaté lors des essais auxquels nous' avons procédé à Liebefeld. Dans une lettre personnelle, Kosıkowski déclara plus tard qu'en mélangeant intimement la chlorophylle avec l'antibiotique, les taches vertes pourraient être supprimées dans le lait. OBIGER [16] a constaté, lors des essais qu'il a effectués avec de la chlorophylle soluble dans l'eau, que s'il en ajoutait $250 \mathrm{mg}$ par essai, la coloration demeurait reconnaissable à l'œil nu jusqu'à la troisième traite (un jour et demi). Après deux jours et demi, on percevait toujours des traces de chlorophylle dans la crème.

\subsection{Essais au moyen de colorants dérivés du triphénylméthane.}

Dans ce groupe de colorants, les substances suivantes ont été soumises à un examen en vue de déterminer si elles pouvaient être ajoutées à des antibiotiques :

a) Vert de lissamine (Food green $\mathrm{N}^{r} 4$; New Colour Index (NCI) $\mathrm{N}^{\mathrm{r}} 44090=$ Green $\mathrm{S}=$ Edicol Supra Green BS = Acid Green $=$ Sel de sodium de l'anhydride tétraméthyl-diaminodiphényl B hydroxynaphtyl-carbinoldisulfonique).

b) Bleu acide brillant V. S. (Food blue $\mathrm{N}^{\mathrm{r}} 3$; NCI $\mathrm{N}^{\mathrm{r}} 42045$ (anciennement CI 737) $=$ Edicol Supra Blue VRS $=$ Acid Blue $=$ Sel de sodium de l'anhydride de l'acide tétraéthyl-diaminotriphénylcarbinol disulfonique). 
c) Alphazurine F.G. (Food Blue $\mathrm{N}^{\mathrm{r}} 2$; BCI $\mathrm{N}^{\mathrm{r}} 42090=\mathrm{Sel}$ d'ammonium de l'anhydride de l'acide dibenzyl-diéthyl-diaminotriphenyl-carbinol-trisulfonique).

Pour les trois colorants précités, on note une étroite relation entre la concentration de l'antibiotique éliminé avec le lait (particulièrement de la pénicilline) et celle du colorant après injection intramammaire.

\subsubsection{Essais au moyen de vert de lissamine.}

Différents chercheurs $(1,12,17)$ ont tout d'abord tenté de déterminer la quantité de colorant qu'il y a lieu de mélanger dans diverses préparations avec $100000 \mathrm{U}$ de pénicilline pour maintenir un bon parallélisme entre la coloration du lait et l'élimination de l'antibiotique. On a constaté que l'addition de $25 \mathrm{mg}$ de colorant par essai ne colorait pas le lait suffisamment et que les résultats étaient meilleurs avec $50 \mathrm{mg}$. Avec $100 \mathrm{mg}$, la coloration est plus intense au cours des deux premières traites (après 12 et 24 heures), mais elle ne subsiste pas plus longtemps qu'avec $50 \mathrm{mg}$. D'autres expériences ont permis de constater que le colorant demeure visible à l'œil nu jusqu'à une concentration de 0,1 à $0,2 \mu \mathrm{g} / \mathrm{ml}$ de lait, que pendant huit mois de stockage l'antibiotique ne subit aucun dommage lorsque la température est maintenue à $5^{\circ} \mathrm{C}$, que le tissu glandulaire n'est pas irrité et que la coloration reste visible jusqu'à 0,1 U de pénicilline par $\mathrm{ml}$ de lait. La plus forte concentration de pénicilline dans du lait, n'entraînant pas une coloration visible, a été de $0,06 \mathrm{U}$ de pénicilline par $\mathrm{ml}$. Si l'on utilisait une résine échangeuse d'ions, le colorant demeurait visible jusqu'à $0,002 \mathrm{U}$ de pénicilline par $\mathrm{ml}$ de lait.

Dalgaard, Mikkelsen et Rasmussen [2] ont mis au point à cette fin de petits tubes en matière plastique à travers lesquels ils introduisent à l'aide d'une seringue, $10 \mathrm{ml}$ du lait à examiner,

Pendant six mois, HogH et Rasmussen [13] ont effectué des recherches sur la teneur en antibiotiques des laits provenant de 32 et 71 troupeaux et livrés dans deux centres de ramassage. Dans 63 troupeaux, les animaux avaient subi un traitement avec des antibiotiques. Conformément aux prescriptions, le lait provenant de vaches ayant été traitées ne devrait pas être livré dans les laiteries. Dans 19 troupeaux, on utilisait un antibiotique qui était éliminé par la mamelle dans un laps de temps maximum de 72 heures. Dans 30 troupeaux, on avait utilisé un antibiotique coloré avec le vert de lissamine. Le traitement fut appliqué deux fois à 48 heures d'intervalle. Il a été possible de détecter l'antibiotique dans le lait jusqu'à concurrence de $0,002 \mathrm{U}$ de pénicilline par $\mathrm{ml}$. 
Dans des fermes où de la pénicilline non-colorée fut utilisée, 11,4 p. $100 \mathrm{du}$ lait contenaient, pendant les quatre premiers jours consécutifs au traitement, plus de $0,002 \mathrm{U} / \mathrm{ml}$.

Dans d'autres fermes où on avait employé de la pénicilline colorée, 0,8 p. 100 seulement du lait contenait de la pénicilline. Dans ce dernier cas, la quantité de l'antibiotique était inférieure à $0,007 \mathrm{U} / \mathrm{ml}$ et aucune coloration du lait n'était visible.

\subsubsection{Essais au moyen de bleu acide brillant V.S.}

Shamani $(18,19,20)$ a utilisé ce colorant et non le vert de lissamine lors de ses essais parce que le premier est autorisé aux Etats-Unis pour la coloration des aliments. Il a constaté que, si l'on ajoutait $100 \mathrm{mg}$ de colorant à $100000 \mathrm{U}$ de pénicilline, la coloration demeurait visible aussi longtemps que l'on pouvait déceler la présence de pénicilline dans le lait. Si la quantité de pénicilline était portée à $900000 \mathrm{U}$, l'élimination de l'antibiotique et du colorant était prolongée de trois traites (un jour et demi). D'une manière générale, l'élimination était prolongée lorsqu'il s'agissait de vaches à faible rendement laitier. Dans les tests de dilution au moyen de lait normal, jusqu'à disparition de la coloration visible à l'œil nu, on a pu noter dans le lait les concentrations maximales ci après :

- Pénicilline en suspension aqueuse : 0,05 $\mathrm{U}$ par $\mathrm{ml}$;

- Auréomycine et terramycine : $0,1 \mu \mathrm{g}$ par $\mathrm{ml}$;

- Streptomycine : $0,15 \mu \mathrm{g}$ par $\mathrm{ml}$;

- Polymycine : $0,25 \mu$ par $\mathrm{ml}$.

Il se produisait toujours un changement de coloration du lait lorsqu'on ajoutait de 0,3 à $0,8 \mu \mathrm{g}$ de colorant par $\mathrm{ml}$. Lors des essais effectués au moyen de néothion (néomycine + thiostreptomycine), l'addition de 85 à $100 \mathrm{mg}$ de colorant pour 7 à $10 \mathrm{ml}$ d'antibiotique a permis de constater la présence de celui-ci durant 3 à 5 traites et celle du colorant durant 4 à 6 traites.

\subsubsection{Essais au moyen d'alphazurine F.G.}

DAwson et FEGAN [4] ont fait des expériences avec plusieurs colorants pour déterminer s'ils étaient susceptibles d'être ajoutés à des antibiotiques. Les produits suivants n'ont pas donné de résultats satisfaisants: fluorescéine, sulfanol bleu $2 \mathrm{R}$ (C1 956), erythrocine (CI 773), violet acide 5 BN (CI 698) et rhodamine B (CI 749). Par contre, on a obtenu de bons résultats avec l'alphazurine F.G. sous forme de sel disodique. D'après les auteurs consultés, ce colorant est autorisé pour des aliments autres que le lait 
dans les pays suivants: Australie, Canada, Cuba, République Dominicaine, Finlande, Grèce, Guatémala, Israël, Japon, Norvège, Pérou, Philippines, Union Sud-Africaine, Uruguay et Etats-Unis d'Amérique. Comparé au vert de lissamine, on a constaté une bonne concordance en ce qui concerne les concentrations décelables.

On a réalisé des préparations à l'aide de 100000 et $25000 \mathrm{U}$ de pénicilline et de $0,25,0,125,0,031$ et 0,023 grammes de colorant dans un milieu hydrophobe. 50 à 60 p. 100 de l'antibiotique injecté et 50 à 100 p. 100 du colorant ont été éliminés avec le lait. Le colorant demeuré visible à l'œil $\mathrm{nu}$ correspondait à $0,1 \mathrm{U}$ de pénicilline par $\mathrm{ml}$ de lait. A la suite des essais effectués sur 14 vaches, on a constaté que la coloration subsistait jusqu'à la septième traite avec moins de $0,1 \mathrm{U}$ de pénicilline par ml de lait.

(à suivre.)

\title{
SUPPLEMENT TECHNIQUE
}

\section{L'EMPLOI DE L'ACIDE LACTIQUE DANS LA FABRICATION DES RÉSINES ALKYDES MODIFIÉES}

\author{
par \\ G. GENIN
}

Ingénieur E.P.C.I.

Le terme "alkyde " s'applique à un groupe de résines synthétiques constituées de résines de polyester modifiées par des huiles, et qui résultent par conséquent de la réaction d'un polyalcool, d'un polyacide et d'acides gras. Le mot "alkyd", dans la langue anglosaxone, a été créé en accolant à la première syllabe du mot alcool, le suffixe kyd correspondant à la dernière syllabe du mot acide. Initialement, ce terme a été appliqué d'une façon générale aux esters polymérisés, mais il est réservé aujourd'hui à des polyesters modifiés par les acides gras et utilisés dans la fabrication des vernis.

Actuellement, la plupart des résines alkydes utilisées dans la préparation des vernis sont préparées en partant d'anhydride phtalique comme principal constituant acide, de glycérine et de pentaérythritol comme polyalcools et d'acides gras dérivés des huiles siccatives ou non. Cependant plus récemment, on a ajouté d'autres produits à cette liste, en particulier le triméthyloléthane et le triméthylolpropane parmi les alcools, et les acides isophta- 